\title{
A Study of Bolt Conclusion Evaluation for Ultrasonic Waves Using Scattering Parameter
}

\author{
Makoto Fukuda $^{\mathrm{a},{ }^{*}}$, Motoyuki Takahashi ${ }^{\mathrm{a}}$, Kazuhiko Imano ${ }^{\mathrm{a}}$ \\ ${ }^{a}$ Department of Electrical and Electronic Engineering, \\ Graduate School of Engineering and Resource Sciences, Akita University, \\ 1-1 Tegata Gakuen-Machi, Akita, 010-8502 Japan \\ *Corresponding Author: mfukuda@gipc.akita-u.ac.jp
}

\begin{abstract}
The evaluation method of the bolt conclusion for ultrasonic waves using reflection coefficient $S_{11}$ of scattering parameter was proposed. Ultrasonic waves of 2 $\mathrm{MHz}$ were used because variation of reflection coefficient $S_{11}$ was the greatest. Reflection coefficient $S_{11}$ was decreased as the axial force was increased. Usefulness of the evaluation method of the bolt conclusion using reflection coefficient $S_{11}$ was demonstrated.
\end{abstract}

Keywords: Ultrasonic waves, Bolt conclusions, Scattering parameter, Reflection coefficient, Axial force.

\section{Introduction}

Bolts and nuts are a standard fastener used in the assembly of mechanical structures or consumer electronics. Because broken, slack, or over-tightened bolts can lead to serious accidents, quality control is therefore an important issue. Current inspection methods for measuring bolt axial force include ultrasonic, strain-gauge, and load-cell techniques. However, complex corrections of the measured values are required to obtain accurate measurements of the bolt axial force when using these methods ${ }^{(1)}$. Therefore, there is demand for simple quality-control techniques. Electromagnetic acoustic transducers ${ }^{(2)}$, piezo cables ${ }^{(3)}$, and smart washers using piezoelectric material ${ }^{(4)}$ have been applied to evaluate bolt condition.

Recently, the measurement of nonlinear ultrasonic waves, including second harmonic components, has been applied for nondestructive evaluation ${ }^{(5-13)}$. The second harmonic components are generated by the nonlinear response of plastic deformation, dislocations, closed cracks or the contact acoustic nonlinearity $(\mathrm{CAN})^{(8)}$. These occur when objects are subjected to finite-amplitude ultrasonic waves. When a structure is fastened using a bolt, an axial stretching force is applied to the bolt. The bolt will be plastically deformed and may grow fractures when the axial force exceeds the bolt's elastic yield point. These plastic deformations and fractures can increase the second harmonic components response of the bolt. Moreover, in the interface of the screw threads of the bolt and the nut, the second harmonic ultrasonic waves will be generated by CAN. In our previous paper, the detection of second harmonic components generated from the fastened bolts was performed using ultrasonic waves transmitted and received, which was increased by approximately $10 \mathrm{~dB}$ or more over before and after fastening bolt ${ }^{(14,15)}$. Contact area between the bolt and the nut should be increased as torque was increased.

If the contact area was changed, reflection and transmission coefficient will be changed. In this study, reflection coefficient $\left(S_{11}\right)$ of scattering parameter will be measured and the relation between torque and $S_{11}$ will be discussed. Evaluation of the bolt conclusion using $S_{11}$ will

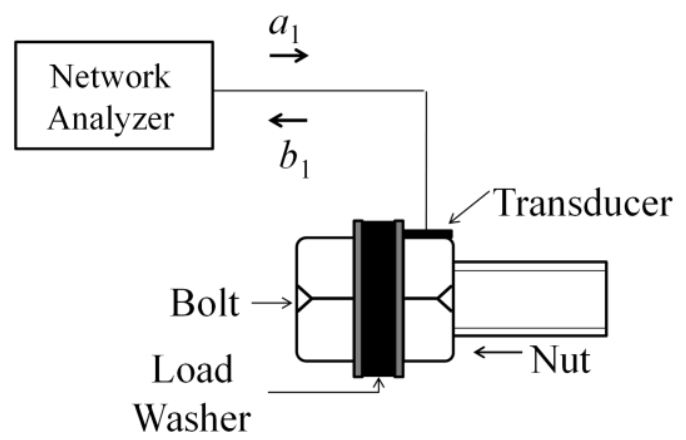

Fig. 1 Experimental set-up. 

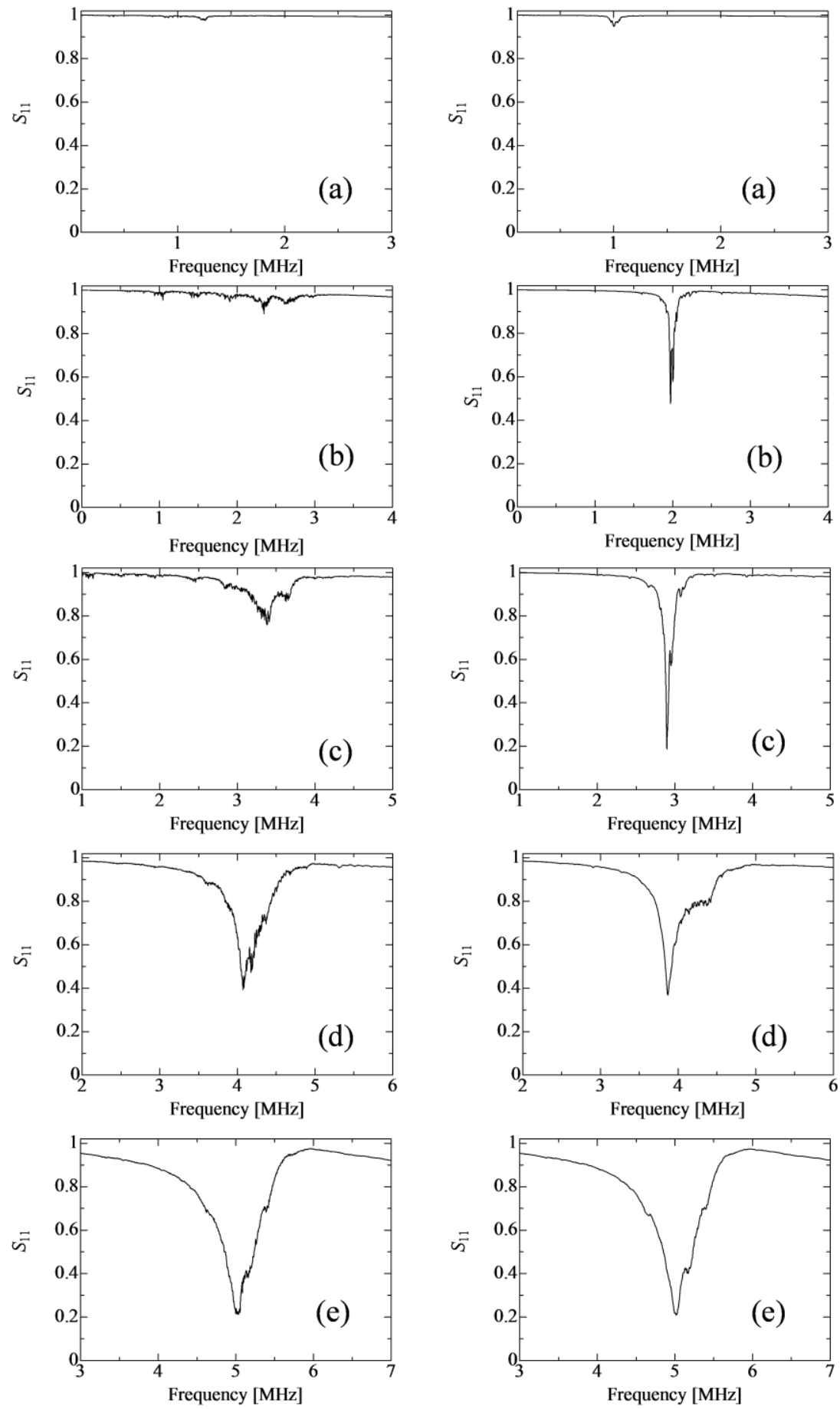

Fig. 2 Reflection coefficient $S_{11}$ when the torque was 0 $\mathrm{N}-\mathrm{m}$. The frequency of ultrasonic waves was (a) 1 $\mathrm{MHz}$, (b) $2 \mathrm{MHz}$, (c) $3 \mathrm{MHz}$, (d) $4 \mathrm{MHz}$ and (e) 5 $\mathrm{MHz}$, respectively.

be studied.

\section{Experimental}

\subsection{Method}

Scattering parameter using two-terminal pair circuit ${ }^{(16)}$

Fig. 3 Reflection coefficient $S_{11}$ when the torque was $50 \mathrm{~N}-\mathrm{m}$. The frequency of ultrasonic waves was (a) $1 \mathrm{MHz}$, (b) $2 \mathrm{MHz}$, (c) $3 \mathrm{MHz}$, (d) 4 $\mathrm{MHz}$ and (e) $5 \mathrm{MHz}$, respectively.

is

$$
\left(\begin{array}{l}
b_{1} \\
b_{2}
\end{array}\right)=\left(\begin{array}{ll}
S_{11} & S_{12} \\
S_{21} & S_{22}
\end{array}\right)\left(\begin{array}{l}
a_{1} \\
a_{2}
\end{array}\right)
$$

where $a_{1}$ is incident electrical power signal of port $1, b_{1}$ is reflection electrical power signal of port $1, a_{2}$ is incident electrical power signal of port 2 and $b_{2}$ is reflection 


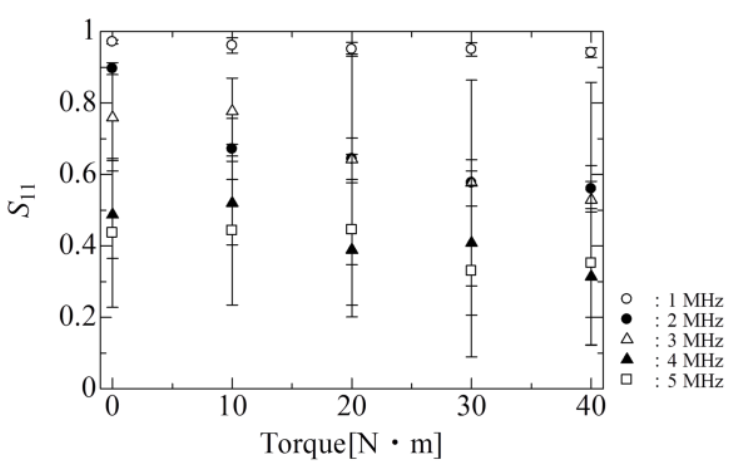

Fig. 4 Relation between torque and $S_{11}$.

electrical power signal of port 2.Reflection coefficient $S_{11}$ is

$$
\left|S_{11}\right|=\left|\frac{b_{1}}{a_{1}}\right| \text {. }
$$

A schematic of our system for detecting the scattering parameter using continuous ultrasonic waves is shown in Fig. 1. Ultrasonic waves are transmitted and received by piezoelectric transducer $\left(\mathrm{PbTiO}_{3}\right)$. The piezoelectric transducer is bonded to an iron bolt-nut conclusion using phenyl salicylate. Reflection coefficient $S_{11}$ will be measured by the network analyzer as the torque or the axial force varied. Torque will be applied by a digital torque wrench. Axial force will be measured by a load washer.

\subsection{Decision of Frequency}

Reflection coefficient $S_{11}$ for the torque when the frequencies are $1-5 \mathrm{MHz}$ is shown in Fig. 2, Fig. 3 and Fig. 4. In the case of 2 and $3 \mathrm{MHz}$, reflection coefficient $S_{11}$ was changed as the torque was increased. On the other hand, in the case of 1,4 and $5 \mathrm{MHz}$, variation of reflection coefficient $S_{11}$ was not confirmed. In $1 \mathrm{MHz}$, since the wave length coincides with the nut length, ultrasonic waves should be diffracted at contacting area. In higher frequencies 4 and $5 \mathrm{MHz}$, attenuation and directionality, which are greater compared with higher frequency, affected ultrasonic waves. In this study, $2 \mathrm{MHz}$ was decided because variation of reflection coefficient $S_{11}$ was the greatest.

\subsection{Results and Discussion}

Reflection coefficient $S_{11}$ for the axial force is shown in Fig. 5 and Fig. 6. Reflection coefficient $S_{11}$ was decreased as the axial force was increased. Most of ultrasonic waves should be reflected the interface between the bolt and the nut. Decrement of reflection coefficient $S_{11}$ corresponds to decrement of the power reflection between the bolt and the nut. The normal axial force of this bolt was $17.5 \mathrm{kN}^{(17)}$. Asperity of contacting surfaces should be planer as the axial force was increased, so reflection coefficient $S_{11}$ was greatly decreased at the closer normal axial force.

Thus, usefulness of the evaluation method of the bolt conclusion using reflection coefficient $S_{11}$ was demonstrated.

\section{Conclusions}

The evaluation method of the bolt conclusion using reflection coefficient $S_{11}$ was proposed. Reflection coefficient $S_{11}$ was decreased as the axial force was increased. Possibility of the evaluation method of the bolt conclusion using reflection coefficient $S_{11}$ was demonstrated.

In the practical use, seal material is used between the bolt and the nut, and/or, several material bolt and nut were used. In the future, possibility in the practical use using our method and application field will be discussed.

\section{Acknowledgment}

This work was supported by a Grant-in-Aid for Young Scientists (B) (No. 25820170) from the Japan Society for the Promotion of Science, and an Academic Grant-in-Aid (H26) from Graduate School of Engineering and Resource Sciences, Akita University.

\section{References}

(1) T. Sakai : "Zouho Neji Teiketsu Gairon (Introduction to Bolt Fastening)", Yokendo, Tokyo, 2005

(2) H. Ogi, M. Hirao and H. Yasui : "Ultrasonic Measurement of Bolt-Axial Stress by A Shear-Wave Electromagnetic Acoustic Transducer", Hihakai Kensa, Vol. 47, No. 5, pp. 331-336, (1998) (in Japanese)

(3) S. Kurosaki, Y. Sasaki and S. Izumi : "Trial of Measurements for Axial Force of Bolt Using Piezo Cable", Hihakai Kensa, Vol. 56, No. 7, pp. 149-154, (2007) (in Japanese)

(4) M. Okugawa and K. Egawa : "Study on Smart Washer Using Piezoelectric Material for Bolt Loosening Detection”, Hihakai Kensa, Vol. 52, No. 9, pp. 511-516, (2003) (in Japanese)

(5) K. Kawashima : "Harmonic Imaging of Plastic Deformation in Thin Metal Plates Using Nonlinear 

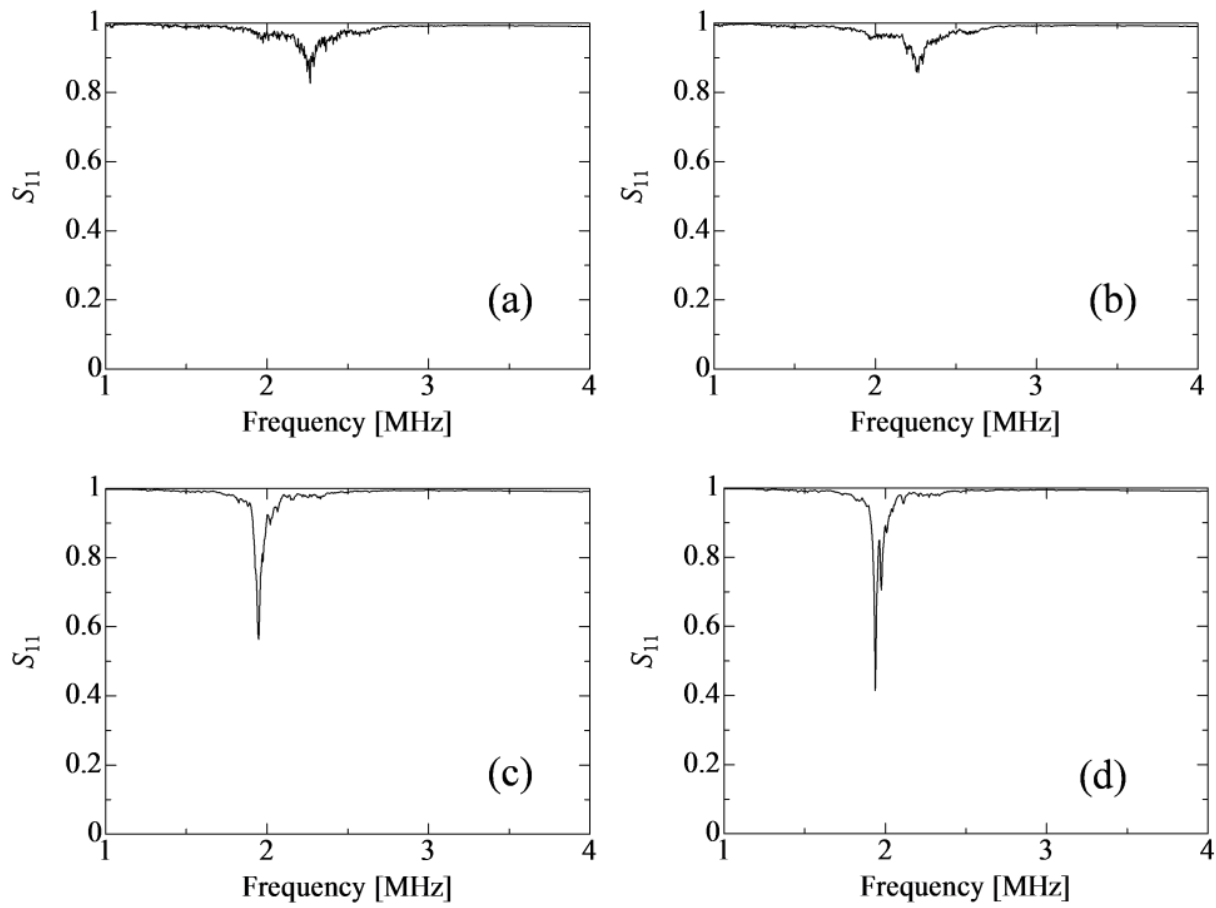

Fig. 5 Reflection coefficient $S_{11}$ when the frequency of ultrasonic waves was $2 \mathrm{MHz}$. The torque was (a) $0 \mathrm{~N}-\mathrm{m}$, (b) $10 \mathrm{~N}-\mathrm{m}$, (c) $20 \mathrm{~N}-\mathrm{m}$ and (d) $30 \mathrm{~N}-\mathrm{m}$, respectively.

Ultrasonic Method", Jpn. J. Appl. Phys., Vol. 50, No. 7, pp.07HC14-1-2, (2011)

(6) M. Ikeuchi, K. Jinno, Y. Ohara and K. Yamanaka : "Improvement of Closed Crack Selectivity in Nonlinear Ultrasonic Imaging Using Fundamental Wave Amplitude Difference", Jpn. J. Appl. Phys., Vol. 52, No. 7, pp.07HC08-1-5, (2013)

(7) S. Biwa, S. Hiraiwa and E. Matsumoto : "Experimental and theoretical study of harmonic generation at contacting interface”, Ultrasonics, Vol. 44, No. Suppl., pp.e1319-e1322, (2006)

(8) I. Y. Solodov, N. Krohn and G. Busse : "CAN: an example of nonclassical acoustic nonlinearity in solids", Ultrasonics, Vol. 40, No. 1-8, pp.621-625, (2004)

(9) M. Fukuda, M. Nishihira and K. Imano : "Real Time Detection of Second-Harmonic Components Generated from Plastic-Deformed Metal Rod Using Double-Layered Piezoelectric Transducer", Jpn. J. Appl. Phys., Vol. 46, No. 7B, pp.4529-4531, (2007)

(10) K. Imano and A. Muto : "Detection of Second Harmonic Wave from the Closed Crack Using Finite amplitude Ultrasonic Wave", J. Soc. Mater. Eng. Resource. Jpn., Vol. 20, No. 1, pp.12-18, (2007) (in Japanese)

(11) M. Fukuda, K. Imano, H. Yamagishi and K. Sasaki :

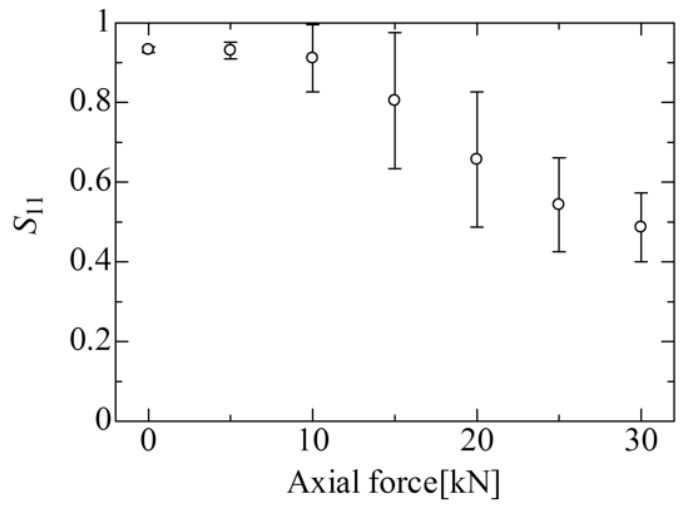

Fig.6 Relation between axial force and $S_{11}$, where ultrasonic frequency was $2 \mathrm{MHz}$.

"Detection of the second harmonics of Lamb waves in fatigued magnesium plates", Acoust. Sci. \& Tech., Vol. 32, No. 6, pp.271-275, (2011)

(12) M. Fukuda and K. Imano : "Detection of Second-Harmonic Components of Lamb Waves Generated from Fatigue Plate Using a Double-Layered Piezoelectric Transducer", Jpn. J. Appl. Phys., Vol. 51, No. 7, pp.07GB06-1-5, (2012)

(13) K. Imano and N. Ishizuka : "Experimental Study of Harmonic Component Generation from the Contact of 
Solids in Ultrasonic Instrumentation", J. Soc. Mater. Eng. Resource. Jpn., Vol. 24, No. 1-2, pp.13-19, (2012) (in Japanese)

(14) M. Fukuda and K. Imano : "Detection of a second harmonic component generated from a fastened bolt using a double-layered piezoelectric transducer", IEICE Electro. Exp., Vol. 6, No. 20, pp.1438-1443, (2009)

(15) M. Fukuda, O. Yonai and K. Imano : "A Study of Bolt Fastening Evaluation Using Increase of Second
Harmonic Ultrasonic Waves against Axial Force", IEICE A, Vol. J96-A, No. 8, pp. 590-592, 2013 (in Japanese)

(16) M. Nakajima : "Micro-ha Kogaku -Kizo to Genri(Microwave Engineeing -Fundamental and Principle-)", Morikita, Tokyo, 1975.

(17) Tohnichi (ed.) : "Tohnichi Torque Handbook Vol.7”, Tohnich Inc., Tokyo, 2008. 